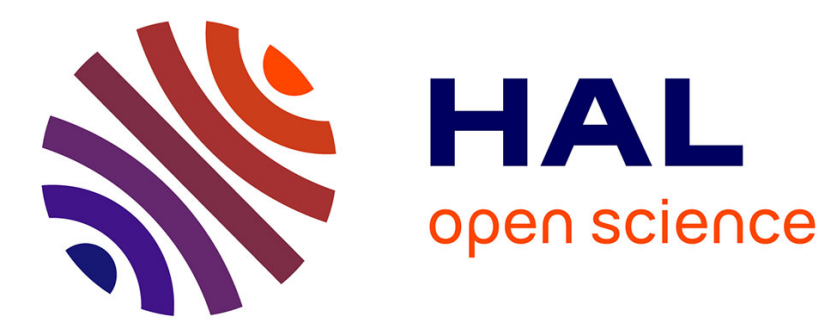

\title{
La pluralité des mondes. Entre science et théologie.
}

Jacques Fantino

\section{To cite this version:}

Jacques Fantino. La pluralité des mondes. Entre science et théologie.. Revue des Sciences Religieuses, 2002, 76, pp.271 - 295. 10.3406/rscir.2002.3629 . hal-01389789

\section{HAL Id: hal-01389789 \\ https://hal.science/hal-01389789}

Submitted on 29 Oct 2016

HAL is a multi-disciplinary open access archive for the deposit and dissemination of scientific research documents, whether they are published or not. The documents may come from teaching and research institutions in France or abroad, or from public or private research centers.
L'archive ouverte pluridisciplinaire HAL, est destinée au dépôt et à la diffusion de documents scientifiques de niveau recherche, publiés ou non, émanant des établissements d'enseignement et de recherche français ou étrangers, des laboratoires publics ou privés. 


\section{La pluralité des mondes. Entre science et théologie}

\section{Jacques Fantino}

\section{Abstract}

The plurality of the worlds is a recurrent question in the Western Tradition since the time of Greeks. It also shows what signifies to know at a time or for an author and what is the specificity of the knowledge in science and in theology.

\section{Résumé}

La pluralité des mondes, question récurrente en Occident depuis les Grecs, est aussi révélatrice de ce que, à une époque ou pour un auteur, connaître veut dire et de ce qui constitue la spécificité de la connaissance en science et en théologie.

\section{Citer ce document / Cite this document :}

Fantino Jacques. La pluralité des mondes. Entre science et théologie. In: Revue des Sciences Religieuses, tome 76, fascicule 3, 2002. pp. 271-295;

doi : 10.3406/rscir.2002.3629

http://www.persee.fr/doc/rscir_0035-2217_2002_num_76_3_3629

Document généré le 03/06/2016 
Revue des sciences religieuses 76 n" 3 (2002), p. 271-295

\section{LA PLURALITÉ DES MONDES, ENTRE SCIENCE ET THÉOLOGIE}

Étudier la question de la pluralité des mondes peut surprendre. Pourtant, savoir s'il existe un seul monde ou plusieurs n'est pas une interrogation nouvelle, mais une problématique récurrente en Occident depuis les Grecs. Actuellement, la question relève plutôt de la littérature de science-fiction et des médias (cinéma et télévision), de sciences comme la cosmologie et de certaines théorisations de la physique quantique, de la logique et de la philosophie du langage. La multiplicité des mondes occupe ainsi une place significative dans la culture contemporaine, mais les mondes envisagés en science, en philosophie et dans les arts sont différents. Logique et philosophie du langage s'intéressent aux univers de langages. Les sciences et les arts sont au contraire préoccupés de mondes « réels" ou supposés tels.

L'universalité du phénomène dans la culture tout autant que la diversité de conceptions des mondes - relèvent-ils de l'imagination humaine, du réel ou bien des deux ? - invitent à préciser le rapport entre ce qu'on entend par réel, la connaissance qu'on en a et la manière dont on en parle. La question de la pluralité des mondes demande donc de réfléchir à deux problèmes corrélatifs. Le premier a trait à ce que veut dire " connaître ", le second concerne la nature de la science par rapport à celles de la philosophie et de la théologie. La première réflexion conduit à élaborer une théorie de la connaissance, une noétique, la seconde construit une théorie du savoir scientifique, une épistémologie. Ce double travail sur ce que penser veut dire et sur les différents types de rationalité remonte à l'Antiquité. Mais, au Moyen Âge en Occident il prend un élan nouveau qui sera transmis à la culture moderne. Au Moyen Âge la théologie a été très impliquée dans le débat sur la pluralité des mondes qu'en fait elle dirigeait. Le débat médiéval s'effectue à nouveaux frais par rapport à celui qui eut lieu dans l'Antiquité du fait du double héritage dont est tributaire l'Occident médiéval : la révélation biblique juive et chrétienne et la culture gréco-latine. 
La question de la pluralité des mondes au Moyen Âge se situe en effet à leur confluent. D'une part, elle se pose dans le cadre de la doctrine biblique de la création et de son explicitation théologique. D'autre part, la méthode théologique, le langage de l'être et de la nature proviennent de la culture gréco-latine où la question de l'existence d'un seul monde ou de plusieurs avait déjà été posée. Cependant, si la prise en compte de la révélation a entraîné des déplacements épistémologiques, nous verrons que sur le fond l'enjeu noétique n'a pas connu le même renouvellement. Si l'argumentation en faveur d'un seul ou de plusieurs mondes varie, la réponse reste fondée sur les mêmes choix théoriques concernant la nature de la connaissance.

\section{LE DÉBAT ANTIQUE SUR LE MONDE}

La philosophie grecque a largement développé la thèse de l'unicité du monde. Seuls quelques philosophes ont avancé l'idée d'une pluralité d'univers. Parmi les Présocratiques, dans l'état actuel des fragments authentiques, il est peut-être possible qu'Anaxagore ait professé cette doctrine (1), mais cela paraît peu probable, car les principes de son système philosophique n'impliquent pas une pluralité des mondes à la différence de ceux des Atomistes (2). En fait, on ne trouve de manière certaine que les Atomistes Leucippe et Démocrite (3) dont les conceptions sont reprises par Épicure et son école et adaptées en fonction des critiques soulevées par les opposants à la théorie atomiste, en particulier Aristote (4).

Mais, ce qui est intéressant ce sont, avec leur fondement, les arguments qui sont développés en faveur de l'unicité ou de la pluralité. C'est pourquoi, deux philosophes sont retenus ici parce qu'ils tiennent des thèses opposées. Il s'agit d'Aristote et d'Épicure. Le premier affirme l'unicité de l'univers, le second la pluralité des mondes. Quelles sont les raisons qui ont poussé ses deux penseurs à soutenir des conceptions du monde aussi opposées l'une à l'autre?

(1) J.-P. Dumont, Éléments d'histoire de la philosophie grecque, Paris, Nathan, 1993, p. 133.

(2) G.S. KIRK, J.E. RAVEN, M. SCHOFIELD, Les philosophes présocratiques. Une histoire critique avec un choix de textes, Fribourg/Paris, Editions Universitaires/Cerf, 1995, p. 406-407. Quant à l'opinion selon laquelle Anaximandre aurait enseigné la pluralité des mondes, elle provient d'une erreur d'interprétation de Théophraste reprise par les commentateurs ultérieurs (ibidem, p. 127-132) et par les chercheurs modernes qui les suivent, telle C. Ramnoux (in B. Parain (éd.), Histoire de la philosophie, 1, Orient, Antiquité, Moyen Âge, Paris, Gallimard, 1969, p. 417-418).

(3) Voir Kirk, Raven, Schofield, op. cit., p. 447-452.

(4) Voir ÉPICURE, Lettres, Maximes, Sentences, traduction, introduction et commentaires par J.-F. BALAUDE, Paris, Librairie Générale Française, 1994, p. 16. 


\section{Aristote et l'unicité du monde}

Selon Aristote monde et nature désignent la totalité de ce qui est, c'est-à-dire l'être. C'est aussi un des sens du terme ciel qui peut encore signifier le monde céleste par opposition à la terre et à l'atmosphère, voire le dernier orbe céleste, le plus éloigné de la terre, selon la cosmologie géocentrique de l'époque.

Aristote s'oppose à la multiplicité réelle et même possible des mondes soutenue par certains philosophes (De caelo A, 8-9, 276a279a). Or, la thèse de la pluralité des mondes repose selon Aristote sur l'existence de l'infini et du vide. Pour cette raison, Aristote critique ces deux idées à partir de ce qui est pour lui un présupposé nécessaire, la théorie du mouvement des corps (Physica $\Gamma, 1,200 \mathrm{~b}$ ). $\mathrm{Au}$ premier plan de celle-ci figurent les notions de nature comme principe de transformation, de mouvement et de corps. Dans cette ligne Aristote se demande s'il existe un corps d'extension infinie. L'existence d'un tel corps impliquerait celle d'une infinité de mondes (Physica $\Gamma, 4,203 \mathrm{~b}$ ). De plus, s'il y a un corps infini, il aura une masse infinie, puisqu'il y a une corrélation entre masse et extension. Pour Aristote, ce n'est pas possible (De caelo A, 5-7, 271b-276a) (5). Le monde est fini. Mais, montrer que l'univers est fini n'implique pas pour autant l'unicité du monde. Le Tout pourrait en effet être constitué d'un nombre fini de mondes différents. Pour cela il faut montrer l'inexistence du vide, car des mondes différents seraient séparés par du vide. Aristote construit sa réflexion sur le vide à partir de sa compréhension du lieu. Un lieu est l'enveloppe d'un corps (Physica $\Delta, 4,212 \mathrm{a}$ ). Il peut être considéré indépendamment du corps, mais on comprend qu'il ne saurait exister de vide au sens absolu, mais seulement dans un sens relatif. En effet, le vide, lieu de ce qui peut être occupé par un corps, est toujours relatif à un corps et ne possède pas de consistance. Il n'est pas une substance (Physica $\Delta, 9$, $217 \mathrm{~b}$ ). Ayant écarté la possibilité de l'existence de l'infini et du vide, Aristote peut alors traiter la question de savoir si le monde est un.

Aristote montre l'unicité du monde d'abord par des arguments physiques. Un corps simple, tel un élément, a un mouvement naturel, c'est-à-dire non contraint, unique et limité (il ne peut être infini). Il tend vers son lieu naturel pour y demeurer. Par exemple, un corps lourd tend vers le centre du monde, c'est-à-dire vers la terre. Or, une pluralité de mondes supposerait une pluralité de centres. Un corps lourd, laissé à son mouvement naturel, doit se diriger vers son lieu

(5) Voir ARistore, Du Ciel, texte établi et traduit par P. Moraux, Coll. Des Universités de France, Paris, Les Belles Lettres, 1965, p. Ix-lxviii ; J.-F. MATTÉI, in J.-F. Mattéi (éd.), Encyclopédie Philosophique Universelle, 3, Les CEuvres Philosophiques, 1, Philosophie Occidentale, Paris, PUF, 1992, p. 50. 
naturel, c'est-à-dire le centre. Mais, son mouvement serait forcé, car il existerait dans cette hypothèse plusieurs centres. Il ne peut donc en exister qu'un seul ( $D e$ caelo A, 8, 276ab). La théorie du mouvement s'oppose ainsi à l'existence de plusieurs mondes.

Aristote raisonne ensuite à partir de la forme en soi ou quiddité d'un être concret, sensible, qui est composé de matière et de forme, c'est-à-dire d'une forme engagée dans la matière. On peut prendre comme exemple la forme du cercle en soi distincte de celle du cercle matériel. Il est alors possible d'appliquer cette distinction au ciel puisqu'il est sensible. La forme du ciel en soi est distincte de celle du ciel matériel. De même qu'il y a pluralité des cercles matériels pour une seule forme en soi, il y aurait pluralité des cieux, et donc des mondes, pour une seule forme en soi du ciel. En fait il n'en est rien, car le ciel épuise la totalité de la matière. Il n'existe pas de pluralité spécifique ou numérique des cieux (De caelo $\mathrm{A}, 8-9,277 \mathrm{~b}-$ $278 \mathrm{~b}$ ). Le ciel et donc le monde est un être individuel, pas une espèce.

Un dernier argument est métaphysique (Metaphysica $\Lambda, 8,1074 a)$. Une pluralité de mondes supposerait pour chacun un premier moteur. Il y aurait un seul principe formel, mais une multiplicité numérique des premiers moteurs. Mais, une multiplicité numérique se fait par la matière. Comme le premier moteur est acte pur donc immatériel, c'est impossible.

À lire les lignes qui précèdent, le monde apparaît comme une structure ordonnée, organique, reflétant la causalité de la nature (Physica $\Lambda, 1,252 \mathrm{a}$ ) et donc son intelligibilité. Le cosmos est à la fois un et unique, traduisant ainsi la solidarité entre la cosmologie et la philosophie première (théologie) : il n'y a qu'un seul principe aux êtres et donc au monde, la divinité encore appelée premier moteur et constituant le dernier ciel (6). Dans cette perspective, il n'y a pas de création. Le monde est éternel incluant le premier moteur qui est d'ailleurs de la même nature, l'éther, que les cieux et les astres.

\section{Épicure et la pluralité des mondes}

Épicure cherche une compréhension du Tout, c'est-à-dire de la totalité de l'être, qui soit la plus compatible avec les phénomènes qui sont visibles ou accessibles à l'être humain. Car, la connaissance est fondée sur la sensation (7). Elle s'obtient en formant des opinions droites (justes) à partir de l'expérience accumulée grâce aux sens. On pro-

(6) Voir P. Merlan, «Greek Philkosophy from Plato to Plotinus », in A.H. Armstrong (ed.), The Cambridge History of Later Greek and Early Medieval Philosophy, Cambridge, Cambridge UP, $1995^{5}$, p. 40-43.

(7) Voir G. ArIGHettı, in B. PARAIN (éd.), Histoire de la philusophie, 1, p. 754755 ; Dumont, p. 476. 
cède de proche en proche, depuis le plus immédiatement accessible aux sens jusqu'au moins accessible, puis aux idées et aux notions. Quand une opinion est confirmée, elle est alors établie et on ne peut l'infirmer, c'est-à-dire soutenir le contraire. En revanche, une opinion qui ne peut être infirmée est considérée par Épicure comme confirmée, c'est-à-dire possible au même titre que d'autres. Il peut en exister plusieurs. En privilégier une au détriment des autres c'est, dit Épicure, sortir du domaine de l'étude de la nature pour se précipiter dans le mythe (Lettre à Pythoclès, 87). La certitude procurée par les sens ne peut être atteinte que pour les réalités proches. Au-delà, on obtient seulement des possibilités compatibles avec les connaissances de ce qui est proche. D'où, la pluralité des explications possibles et l'impossiblité de tenir un discours unique, c'est-à-dire de renvoyer à un principe unique (8). Épicure rejette tout principe ontologique posé au départ, car cela revient pour lui à poser un acte de foi. La connaissance est à la fois en genèse, elle se développe selon un processus de formation, et empirique, elle part de l'expérience sensible accumulée et organisée.

Dans cette perspective les phénomènes cosmiques non accessibles aux sens de manière adéquate, à la différence de ceux de notre environnement proche, font que l'on est réduit à leur sujet à des conjectures, œuvres de la pensée. Des hypothèses différentes, voire contradictoires peuvent alors être soutenues. C'est le cas par exemple pour expliquer les mouvements du soleil et de la lune (Lettre à Pythoclès, 93). Dans cette perspective la thèse de la multiplicité des mondes résulte de l'impossibilité d'affirmer d'une manière probante l'existence d'un monde unique. Explicitons la démarche d'Épicure concernant la connaissance de l'univers.

La connaissance, fruit de l'expérience ordinaire, montre en premier que tout est constitué de corps. Les corps présentent deux propriétés fondamentales. D'une part, ils sont en mouvement permanent : astres, êtres vivants, éléments, etc. Le mouvement n'a ni commencement, ni fin (9). D'autre part, les corps n'existent pas indéfiniment. Ils se désagrègent en unités de matière plus petites tandis que d'autres corps apparaissent par agrégats d'unités de matières. Les unités les plus élémentaires, et par conséquent indestructibles, sont les atomes. Les corps sont des agrégats temporaires d'atomes. L'existence du mouvement implique enfin celle du vide entre les corps pour permettre justement leurs déplacements.

Finalement, comme le Tout est la totalité de l'être, il est alors constitué de corps et de vide. Or, l'observation montre qu'il ne pos-

(8) Voir Balaudé, op. cit., p. 106-107

(9) Ibid., p. 63. 
sède pas de limite visible. Épicure en tire comme conséquence que le Tout est illimité, infini, tout comme le nombre des corps ainsi que l'extension du vide (Lettre à Hérodote, 41-42) (10).

En revanche, à la différence du Tout, l'expérience révèle encore

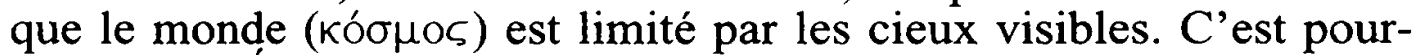
quoi, selon Épicure, le monde et le Tout ne désignent pas des réalités identiques. De plus, le monde est ordonné. L'ordre ne peut exister que dans des domaines limités. Le Tout n'est donc pas ordonné.

De cela résulte que l'ordre, c'est-à-dire un ensemble ordonné de corps en mouvement, est limité et qu'il en existe une pluralité au sein du Tout qui, lui, est unique (11). La totalité ne peut être ordonnée, sans doute à cause du mouvement permanent qui produit l'agrégation et la désagrégation des atomes. Les mondes se construisent à partir d'amas de matière (d'atomes) qui constituent l'intermédiaire entre le vide et eux. Néanmoins, s'il y a une pluralité de mondes, il n'y a cependant pas une infinité de configurations possibles (Lettre à Hérodote, 74). La démarche d'Épicure conduit à une position inverse de celle d'Aristote. Comme l'exposé l'a montré le système épicurien part d'une théorie de la connaissance qui engage une conception de la science pour traiter en un second temps de la question du Tout. $\mathrm{Ne}$ serait-ce pas cette noétique et cette épistémologie qui guideraient Épicure vers la thèse de la pluralité des mondes? Mais alors n'en serait-il pas de même chez Aristote?

\section{Les enjeux noétique et épistémologique du débat}

Pour Épicure, l'impossibilité de réduire la multiplicité des phénomènes à l'unité, c'est-à-dire à un seul principe explicatif, tient à la situation de l'être humain et aux limites de son intelligence. Pour ce qui n'est pas directement accessible, l'intelligence, faute de mieux, est contrainte de tenir une pluralité d'explications possibles compatibles avec ce qui est mieux connu (12). Savoir si le Tout est constitué d'un seul monde ou de plusieurs est certes une question ontologique, mais la réponse est tributaire d'une noétique et d'une épistémologie. Ce n'est pas la question de la pluralité des mondes en tant que telle qui suscite cette remarque, cela est vrai de tout autre question ontologique, mais celle-ci par la réponse donnée, un seul univers ou plusieurs, fait apparaître de manière éclatante les enjeux noétique et épistémologique.

(10) Voir Dumont, op. cit., p. 478

(11) Voir ARIGHITTI, op. cit., p. 766 ; BALAUdE, op. cit., p. 74.

(12) A.A. Lons, D.N. Stedly, Les philosophies hellénistiques, 1, Pyrrhon, L'épicurisme, trad. de J. Brunschwig et P. Pellegrin, Paris, Flammarion, 2001, p. 195198. 
La réponse épicurienne affirmant la pluralité des mondes est liée à l'esprit humain cherchant à comprendre ou à situer son existence dans l'ensemble de ce qui existe : Notre mode de vie n'a pas besoin de théories personnelles ni d'opinions vides, mais de vivre sans trouble (Lettre à Pythoclès, 87). Ce résultat est atteint quand on garde la multiplicité des explications en accord avec ce qui apparaît (ibidem).

Cette conclusion vaut pour la démarche d'Aristote. Chez lui aussi la connaissance part du concret et donc du sensible, mais en même temps elle vise l'universel. C'est pourquoi la connaissance est à la fois empirique et abstraite. Elle procède d'une part de la sensation. de la mémoire et de l'expérience. D'autre part, la connaissance en tant que science se vérifie par voie déductive à partir des principes, démarche qui préserve ainsi le savoir des erreurs. Toute science repose sur la démonstration à partir de principes vrais (Analytica posteriora, $1,2,71 b)(13)$. Ces premiers principes ne sont connus par l'intellect que par l'intuition : Des principes, il n'y aura pas de science [...] C'est une intuition qui appréhendera les principes [...] C'est l'intuition qui sera principe de la science (Analytica posteriora, 2, 19, 100b). Et l'intuition repose sur l'induction à partir des particuliers (14). L'intellection inductive est ainsi au dessus de la science, au sens où elle la fonde en procurant l'évidence des premiers principes.

Maintenant que la science est définie, il reste à voir la connaissance du réel qu'elle apporte. La connaissance d'un être concret a pour objet sa forme. La forme est d'abord l'ensemble des caractéristiques générales qui sont communes à tous les êtres d'une même espèce. Elle est ensuite l'ensemble des déterminations particulières qui différencient un individu d'un autre de la même espèce. Connaître un être, c'est d'abord connaître ses caractéristiques générales qui permettent de dire ce qu'il est avant de le distinguer par des déterminations particulières. La connaissance va donc du général au particulier. Toutes les sciences visent par conséquent le général et sont classées selon leur degré de généralité qui dépend des principes premiers de chacune. L'ontologie repose sur une noétique selon laquelle les formes constituent les objets de la connaissance et sur une épistémologie selon laquelle une science procède par démonstration à partir de principes.

Aristote et Épicure partent donc du même point de départ, le réel tel qu'il est perçu. A partir de ce point commun ils organisent néanmoins leur réflexion selon deux perspectives opposées. Pour Aristote, la connaissance est générale et globale. Elle embrasse le réel dans sa

(13) Voir P. Pell.egris, in J.-F. MattF́ (éd.), Encyclopédie Philosophique Universelle, 3, p. 46.

(14) Ibid. 
totalité. Épicure, au contraire, privilégie la connaissance proche, celle du particulier et du local. Ces deux noétiques différentes, que j'appellerai respectivement globale et locale, engendrent des épistémologies et des ontologies, c'est-à-dire des compréhensions de la science et de l'univers, qui sont opposées.

Une connaissance de type global, comme chez Aristote, conduit nécessairement à affirmer l'unicité du monde. Toute science va du général au particulier et c'est pourquoi Aristote analyse le réel selon un ordre bien précis. Il commence par étudier les principes du mouvement, puis l'agencement des mouvements dans l'univers pour terminer par ceux des étants naturels. Après avoir défini la science naturelle, la physique, comme science du mouvement, et comme le remarque fort justement P. Pellegrin, Aristote s'intéresse au mouvement céleste, qui est le mouvement premier auquel tous les autres en fin de compte se ramènent (15).

La connaissance de type local telle que la pratique Épicure aboutit au contraire à affirmer la pluralité des mondes. Ce résultat est bien en cohérence avec la noétique et l'épistémologie du système épicurien. La connaissance se déploie de proche en proche. La science est d'abord "locale ", puis par des extensions successives elle gagne la totalité de ce qui existe. Ce faisant, plus l'analyse s'éloigne du domaine de l'expérience et donc du savoir local, plus la connaissance dépend d'hypothèses qu'on ne peut prouver mais qui s'accordent à ce qui est déjà connu ou tout au moins n'y contredit pas. Parmi différentes hypothèses possibles, il faut tenir la plus plausible. On peut affirmer l'existence d'un seul monde tout comme de plusieurs sans pouvoir prouver l'un ou l'autre. Mais, le nombre infini des atomes et donc des corps rend plus plausible l'affirmation de la pluralité.

Bien que partant tous deux de l'analyse du réel, Aristote et Épicure en tirent cependant des conceptions radicalement différentes, parce qu'elles dépendent d'une mise en perspective particulière du réel et de la connaissance qu'on peut en avoir. Une dernière remarque est importante. Pour ces deux philosophes, comme pour tous les autres, il y a communauté de langage et de pensée entre science, philosophie et théologie. On passe de l'une à l'autre de manière continue. La réflexion sur les différents domaines du réel s'accompagne d'une réflexion sur leurs principes et sur la connaissance. De même, on passe de l'univers à l'étude du divin. Cette manière de procéder change lors de la reprise de la culture hellénistique par le judaïsme puis par le christianisme qui dans leur réflexion théologique font appel

(15) Voir ARISTOTr, Physique, Introduction et traduction de P. Pellegrin, Paris, Flammarion, p. 26 et $44-49$. 
à une révélation et non plus seulement à la nature. C'est donc dans un cadre culturel modifié que la question de la pluralité des mondes réapparaît au Moyen Âge (16).

\section{LE DÉBAT MÉdIÉVAL SUR LA PUISSANCE DIVINE:}

La question de la pluralité des mondes a rebondi au Moyen Âge à partir du XII' siècle avec l'apport de traductions inédites, comme on va le voir. On se limitera à la période d'environ deux siècles (vers 1130-1330) qui a vu naître et se développer le débat sur cette question.

Le dossier de la pluralité des mondes a été étudié au plan de l'histoire des idées (17), et abordé à un plan plus purement philosophique, avec le renouveau contemporain des études en philosophie médiévale (18). Mais on a peu approfondi les raisons qui expliquent pourquoi cette question a occasionné un tel débat alors que la réflexion sur la puissance divine engage d'autres questions autrement plus importantes tant pour la théologie que pour la philosophie. Comme pour l'Antiquité, derrière le débat médiéval sur la pluralité des mondes, on trouve la trace d'un autre questionnement, épistémologique et noétique, sur ce que l'être humain peut connaître du créé et sur la manière dont il le connaît.

Car, la période étudiée correspond à l'émergence d'une nouvelle manière de faire de la théologie qui va s'imposer comme paradigme pour toutes les autres disciplines avec la création des universités, phénomène qui conditionne le questionnement sur la pluralité des mondes et qui est rappelé dans la première partie de cette étude. L'analyse continue avec l'histoire de cette question dans la période considérée pour traiter enfin, en un troisième temps, ce qui apparaît comme sous-jacent aux réponses contradictoires données par les théologiens à cette question.

\section{Le passage à la théologie systématique}

La théologie vers 1100 consiste essentiellement en un travail de commentaire des Écritures et des Pères. C'est d'ailleurs à ce

(16) Elle fut abordée par les auteurs chrétiens de l'Antiquité et du haut Moyen Âge, mais d'une façon purement scolaire à partir des doxographies, ces compilations d'auteurs, et sans susciter de débat nouveau. Voir J. PéPIN, Théologie cosmique, théologie chrétienne, Paris, PUF, 1964.

(17) P. DuHEM, Le système du monde, 9 vol., Paris, Hermann, 1958 ; S.J. Dick, La pluralité des mondes, Paris, Actes Sud, 1989; E. Grant, Planets, Stars, and Orbs. The medieval cosmos, 1200-1687, Cambridge, Cambridge Univeristy Press, 1996.

(18) Par exemple O. Boulnols, La puissance et son ombre. De Pierre Lombard à Luther, Paris, Aubier, 1994. 
moment qu'est rédigée la Glose ordinaire, commentaire suivi de toute la Bible où le texte biblique était accompagné de citations des Pères le concernant (19). Abélard introduit un changement dans la manière de faire de la théologie. Son but est de mettre en place une lecture technique de la Bible et de la tradition théologique qui s'adresse à l'intelligence plutôt que de nourrir seulement la spiritualité. Il s'agit de faire place à la critique. D'autres théologiens, comme Anselme de Canterbury, avaient déjà initié la théologie comme réflexion critique, mais cc qui est nouveau avec Abélard c'est l'extension de la méthode à tout le travail théologique et pas seulement à quelques questions bien délimitées. Abélard est d'ailleurs le premier à utiliser le terme de théologie pour désigner un ouvrage systématique. C'est une innovation, car le champ théologique n'est plus organisé selon les textes des Écritures et des Pères ou selon une question traitée à partir d'eux. Il est dorénavant organisé par l'intelligence seule (20).

Cette nouvelle manière de faire de la théologie, après des déboires et des excès initiaux, devient peu à peu la règle et trouve sa forme quasi-définitive au milieu du siècle chez Pierre Lombard (21). On sait que maître en théologie, puis évêque de Paris, il a rédigé vers 1155 1158 un ouvrage intitulé Les Sentences. Ce traité parcourt tout le champ théologique de manière systématique. Avec lui la totalité du pensable s'organise selon une finalité universelle. Pierre Lombard reconduit tout l'univers à Dieu, par l'intermédiaire du Christ incarné et des sacrements de l'Église (22). Il s'agit d'organiser les données des Écritures et des Pères selon un ordre nouveau orienté selon les dimensions de l'existence chrétienne qui mène les créatures vers Dieu. Le livre des Sentences de Pierre Lombard (23) devient à partir du $\mathrm{XIII}^{\mathrm{e}}$ siècle le manuel théologique de référence que tout théologien se devait d'avoir expliqué.

Le passage à la théologie systématique correspond à un moment charnière de l'Occident médiéval. Le double héritage dont il est en effet tributaire, à savoir la révélation biblique juive et chrétienne et la culture gréco-latine, s'enrichit d'une manière décisive au XII' siècle. Du premier, la pensée médiévale tient la révélation chrétienne et son

(19) Voir J. Jolivet, La théologie d'Abélard, Paris, Cerf, 1997, p. 22.

(20) Sur la théologie d'Abélard, voir JOLIVET, p. 19-67.

(21) Sur l'origine et la forme de la théologie scolastique, voir par exemple E. VILANova, Histoire des théologies chrétiennes, 1, Des origines au $15^{\circ}$ siècle, Paris, Cerf, 1997, p. 711-721

(22) O. BOULNOIS, p. 14-15.

(23) Magistri Petri Lombard, Sententiae in IV Libris Distinctae, Grottaferrata,

Éd. Collegii S. Bonaventurae Ad Claras Aquas, 1971 ${ }^{3}$. 
explicitation par les Pères et les docteurs. Le second héritage fournit à la théologie son langage et son cadre de pensée. Le premier est augmenté des traductions de plusieurs Pères grecs et le second s'accroît de l'arrivée des œuvres d'Aristote et de ses commentateurs grecs et arabes traduites en latin à cette époque. Non pas qu'Aristote était inconnu auparavant, mais on ne connaissait essentiellement que son ceuvre logique. Avec l'ensemble du corpus qui couvre pratiquement tous les domaines scientifiques et philosophiques, l'Occident possède dès lors une référence pour toutes les connaissances profanes, c'est-à-dire ne faisant pas appel à la révélation judéo-chrétienne. Auparavant science et philosophie étaient davantage des compilations de divers auteurs et écoles. Dorénavant, l'Occident dispose d'un langage et des concepts qui constituent un système de pensée homogène s'étendant à tout le réel.

Avec le système aristotélicien, le Moyen Âge développe une compréhension du réel selon une construction à trois étages élaborée au XII ${ }^{e}$ siècle (24). À la base figure la connaissance rationnelle du réel selon le corpus scientifique d'Aristote, notamment la Physique et le traité $\mathrm{Du}$ ciel. Cette compréhension du monde requiert ensuite, et c'est le deuxième étage de la construction, la connaissance des principes qui structurent le réel, objet de la Métaphysique. Celle-ci est une sorte de théologie naturelle, au sens d'une théologie élaborée uniquement par l'intelligence humaine sans recourir à une révélation. Cette théologie conduit à affirmer l'existence de Dieu comme premier Principe et fait le lien avec la théologie bâtie à l'aide de la révélation. Cette dernière théologie constitue le troisième étage de la construction intellectuelle avec laquelle tout le Moyen Âge occidental va fonctionner. Cette construction est analogue à celle des cathédrales gothiques avec leurs trois ordres superposés (25). On rencontre en premier l'étage des arcades, espace de la terre et des êtres humains, puis au dessus de lui se trouve le triforium qui fait le tour de la cathédrale et assure la jonction entre le haut et le bas, et enfin l'étage des grandes fenêtres, domaine de la lumière qui vient du ciel.

Le fait qu'il y ait une théologie qui ne fasse pas appel à la révélation à côté d'une autre qui y fait appel ne doit pas surprendre. C'est la manière dont le $\mathrm{XII}^{\mathrm{e}}$ siècle traduit à partir du système aristotélicien la doctrine traditionnelle des deux livres de la révélation, la nature

(24) G. SiEgWalt, Dogmatique pour la catholicité évangélique, 3, L'affirmation de la foi, 1, Cosmologie théologique : sciences et philosophie de la nature, Paris/ Genève, Cerf/Labor et Fides, 1996, p. 50-51

(25) Concernant le rapport existant entre architecture gothique et pensée scolastique, voir E. PANOFSKY, Architecture gothique et pensée scolastique, Paris, Les Éditions de Minuit, 1967. 
accessible à tous et les Écritures transmises et interprétées par l'Église. L'explicitation de ces deux livres constitue la science et la philosophie d'un côté et la théologie de l'autre. Ce que l'on sait par les connaissances profanes doit rejoindre l'enseignement tiré de la révélation biblique et le lien est effectué par la philosophie. Remarquons également que c'est la même conception de la connaissance qui est à l'œuvre en science, philosophie et théologie.

Finalement, la nouvelle théologie se caractérise par sa méthode et la définition de son champ d'activité. Par sa méthode, qui est celle du questionnement, la théologie interroge les autorités de la tradition, Ecritures, Pères, conciles, etc. pour approfondir leur compréhension en se servant de leurs oppositions éventuelles ou pour répondre à des questions nouvelles. La théologie organise le champ du questionnement en le répartissant en différents domaines connexes. Ainsi, les questions sur Dieu occupent un de ces domaines, celles sur le créé un autre, et ainsi de suite. Enfin, grâce au système aristotélicien, la théologie est située par rapport aux connaissances profanes, celles qui relèvent de l'exercice de l'intelligence sans recours à la révélation, c'est-à-dire philosophie et science.

\section{Un seul monde ou plusieurs : histoire de la question}

La question de savoir s'il existe un seul monde ou plusieurs est traitée dans le cadre de l'étude de la puissance créatrice de Dieu. La réflexion interprète la révélation biblique et la tradition théologique à l'aide de l'outil méthodologique et conceptuel aristotélicien.

Sur cette question, la révélation est résumée dans la confession de foi en un Dieu Créateur : Nous croyons en un seul Dieu, le Père tout puissant, Créateur du ciel et de la terre, etc. Lors d'une première étape qui va jusque vers 1240 , la réflexion consiste à mettre en évidence que la puissance divine n'est en rien limitée par la création de ce monde-ci.

Le début de la réflexion théologique : un seul monde réel (vers 1140-1240)

$\mathrm{Au} \mathrm{XII}^{\mathrm{e}}$ siècle, en continuité avec l'opinion commune des Pères et des premiers docteurs médiévaux, la théologie interprète l'affirmation biblique concernant l'acte créateur comme signifiant l'existence d'un seul monde, considéré comme étant l'ensemble du créé, c'està-dire la totalité de ce qui existe en dehors de Dieu. La nouveauté de la réflexion théologique tient dans le questionnement porté sur la puissance que le Créateur met en œuvre pour produire ce monde. Abélard soutient que Dieu ne peut faire que ce qu'il fait, enseignement condamné au concile de Sens en 1141. Pierre Lombard reprend la 
question en se demandant si Dieu peut faire autre chose que ce qu'il fait (Sentences 1, d. 43) ou s'il peut faire quelque chose mieux qu'il ne le fait (Sentences 1, d. 44, c. 1). Après un long examen d'opinions contraires Pierre Lombard répond positivement à la première question, à savoir que Dieu peut, sans mutation de sa puissance ou de sa volonté, à la fois vouloir et faire autre chose que ce qu'il veut et fait (d. 43, $\left.\mathrm{n}^{\circ} 10\right)$. De là découle la réponse à l'autre question : si on réfère la modalité de l'opération à la sagesse du Créateur, elle ne peut être ni autre, ni meilleure. Si maintenant on réfère la modalité à la réalité même que Dieu fait, nous disons que la modalité peut être autre ou meilleure... Dieu peut donc faire qu'une réalité qu il fait soit selon une modalité meilleure, une autre selon une modalité également bonne, une autre selon une modalité moins bonne qu'il ne le fait (d. 44, c. 1, n 4).

Ces considérations n'entament pas la conviction commune des théologiens, dont Pierre Lombard, concernant l'unicité du monde.

Dans cette perspective, puisque le livre de la nature est en harmonie avec celui de la révélation, l'affirmation théologique de l'unicité du monde doit être en consonance avec ce que disent la science et la philosophie.

Le discours scientifique sur l'unicité du monde reprend son argumentation à Aristote qui, rappelons-le, est la référence scientifique de l'époque. À sa suite on met en avant l'inexistence du vide (26). De même, la théorie aristotélicienne du mouvement des corps sert également de lieu commun pour prouver l'unicité du monde. Au plan métaphysique deux arguments vont jouer. Le premier est de dire que l'acte de création épuise la matière créée en vue de ce monde-ci et qu'ainsi il n'y en a plus de disponible pour en créer un autre. Le second argument tient dans le fait que selon la démonstration d'Aristote le Principe du monde est unique. Une pluralité de mondes entrâ̂nerait une pluralité de principes. Or, le principe étant immatériel, cela n'est pas possible puisqu'il n'y a de multiplicité que matérielle.

Ainsi est explicitée la relation entre science et théologie, selon le système intellectuel à trois étages décrit plus haut, en montrant le lien entre l'acte créateur et la toute puissance divine.

Les théologiens affirment en effet que Dieu, en créant, a conféré au créé un ordre, une organisation unique qui fait qu'un seul monde réel peut exister. C'est la distinction entre puissance absolue et puissance ordonnée. La première consiste dans la toute puissance de Dieu qui peut tout faire. La puissance ordonnée est celle que Dieu met en

(26) Voir la réflexion aristotélicienne présentée plus haut ainsi que Duhem, t. 9 , Paris, Hermann, 1958, p. 364-369. 
œuvre en réalisant son dessein créateur avec l'organisation du créé que cela suppose. Dieu impose un ordre au créé qui ne peut aller contre lui. C'est dans cette perspective théologique que sont reçus les arguments scientifiques en faveur de l'unicité du monde. L'existence de plusieurs mondes impliquerait celle du vide pour séparer ces mondes. Le vide est certes quelque chose de possible à faire pour le Créateur selon sa puissance absolue, mais pas selon sa puissance ordonnée. Car, celle-ci suppose un ordre substantiel : l'univers est unique comme l'est un individu. Il n'y a pas de place pour le vide, sinon comme relatif à un lieu et à un corps, ainsi que le précisent les théologiens, tel Roger Bacon (Opus Maius, p. 4, d. 4, c. 12). Mais, l'argumentation scientifique constitue seulement un appui à l'interprétation habituelle de la révélation et non une démonstration. On peut ainsi remarquer que l'inexistence du vide est un argument contre la pluralité des mondes, mais qu'il n'y a pas équivalence entre les deux thèses, l'existence du vide et celle de plusieurs mondes (27). De même en est-il pour l'argument selon lequel la matière créée par Dieu est épuisée dans la formation du monde tel que nous le connaissons. L'idée à l'arrière-plan de toutes ces réflexions est que l'unicité du monde relève des limites inhérentes à la nature créée et non de la puissance absolue du Créateur (28). Les contraintes appartiennent au créé pas au Créateur.

Un seul monde réel parmi une infinité de mondes possibles (vers 1240-1277)

La réflexion sur la puissance divine montre que les limites inhérentes au créé ne proviennent pas d'une déficience de cette puissance. Dieu peut faire ce qu'il veut, mais ce qu'il fait est limité par rapport à lui. Il aurait pu aussi faire comme l'a montré Pierre Lombard un monde autre. Mais, que veut dire autre ? Vers le milieu du XIII siècle les théologiens vont s'intéresser à cette question. Pour cela ils partent à nouveau d'Aristote et de la distinction qu'il fait entre la substance qui dans un être constitue ce qui demeure et les accidents qui correspondent à ce qui est susceptible de changement. Deux possibilités existent alors pour comprendre en quoi un monde peut être autre que le nôtre. Il le sera par les substances ou par les accidents.

Dans ce dernier cas on aurait affaire à deux mondes semblables mais numériquement distincts, comme deux individus d'une même espèce. On a alors affaire à un monde autre, altéré accidentellement, c'est-à-dire changé de sorte que par exemple la Lune serait plus grosse et plus éloignée, les espèces vivantes seraient les mêmes, mais leurs

(27) Ce qu'a tendance à faire Duhem, op. cit., t. 8, p. 28-35.

(28) Voir DlHFM, op. cit., t. 9, p. 365-373. 
proportions différentes. Cela ne constitue pas réellement un autre monde au sens strict.

Tout autre serait le cas où Dieu aurait créé selon des conditions telles que cela touche aux substances constituant le monde. C'est tout l'ensemble qui serait alors substantiellement différent, les substances n'étant plus les mêmes. Alors, on aurait véritablement affaire à un autre monde.

Ces deux possibilités conduisent à bien distinguer entre multiplicité et pluralité. Des mondes accidentellement différents constitueraient une multiplicité de mondes, pas une pluralité. Ce serait le cas en revanche de mondes différant par les substances qui les composent. Cette réflexion est partagée par plusieurs théologiens, comme Bonaventure (29). Le monde, on le voit, signifie toujours l'univers au sens de la totalité du créé, c'est-à-dire de ce qui existe autre que Dieu.

Cependant, il reste à déterminer si ces spéculations sur l'existence d'un autre monde que le nôtre constituent seulement une hypothèse ou bien représentent une réalité. C'est là qu'intervient la réflexion sur la puissance divine qui connaît un nouveau développement avec la distinction entre ce qui est en acte, le réel, et ce qui est en puissance, le possible. Dieu a créé un seul monde réel, mais parmi une possibilité infinie d'autres mondes. La distinction entre le possible et le réel recouvre alors celle qui existe entre la puissance absolue du Créateur et sa puissance ordonnée. La puissance absolue, on l'a vu, exprime la toute puissance divine, ce qui est compris ainsi : Dieu peut faire tout excepté ce qui est contradictoire, par exemple faire à la fois qu'une chose existe et n'existe pas (30). La puissance ordonnée correspond alors à une mise en œuvre de la puissance divine selon un ordre déterminé. Quand Dieu crée, il crée selon un ordre, un schéma déterminé. Cela ne veut pas dire que tout existe ipso facto selon le dessein créateur, mais que l'univers est ordonné de manière optimale à tout moment par rapport à sa fin même si cet ordre ne devient parfait que lorsqu'il atteint cette fin (31). Les théologiens maintiennent de la sorte la réponse traditionnelle tout en l'appuyant sur une compréhension plus avancée de la puissance divine.

La pluralité ou la multiplicité des mondes envisagée jusqu'ici par la pensée médiévale demeure une pure spéculation théologique. Elle rejaillit au plan métaphysique, il suffit de se rappeler la construction à trois étages, par la question des possibles. Pour la métaphysique, il n'y a qu'un seul monde réel puisque le terme monde désigne la totalité de l'être créé. Il y a lieu en effet d'articuler au plan des principes

(29) Voir, par exemple, Bonaventurf, Sur les Sentences, 1, d. 44, q. 1.

(30) Thomas D'AQuin, Summa Theologica, p. 1, q. 25, a. 3.

(31) Voir BonaVENTURE, ibid. d. 44 , q. 3 
l'unicité du monde réel à la pluralité des mondes possibles. Mais, cela ne va pas plus loin et n'atteint pas le niveau physique : si l'on conçoit une pluralité de mondes possibles, il existe un seul monde réel. Dans cette ligne, et c'est important de le souligner, la physique d'Aristote est alors perçue comme un cas particulier parmi un ensemble de physiques différentes possibles (32).

Malgré cela, l'argumentation des théologiens en faveur de l'unicité réelle du monde est le plus souvent appuyée par des preuves tirées de la science et de la métaphysique aristotéliciennes. C'est le cas en particulier de l'impossibilité d'un infini en acte. Or, pour le théologien, la toute puissance divine est nécessairement infinie. Cette affirmation ouvre alors la possibilité de concevoir l'existence réelle de plusieurs mondes créés. Mais, ce sont les contraintes rationnelles imposées par le système aristotélicien qui conduisent certains à continuer d'affirmer que Dieu ne peut pas créer plusieurs mondes au nom même de la raison. On comprend alors la réaction qui aboutit en 1277 à la condamnation par Étienne Tempier, alors évêque de Paris, de plusieurs propositions couramment débattues. Cette intervention vise en fait à libérer la théologie du nécessitarisme aristotélicien. Parmi les énoncés condamnés en figure un qui concerne directement la question de la pluralité des mondes. Il s'agit de la thèse 34 selon laquelle la cause première ne pouvait pas faire plusieurs mondes.

Le renversement de la problématique (1277-vers 1340)

Les théologiens doivent dorénavant enseigner la possibilité, et non plus l'impossibilité, pour Dieu de créer plusieurs mondes et surtout éviter de montrer l'unicité du monde à partir seulement de la science et de la philosophie d'Aristote. La réflexion sur la toute puissance divine et la liberté qui lui est associée va détourner l'attention de la compréhension du réel vers l'analyse du possible, de ce qui est donné par la puissance ordonnée vers ce que dans sa puissance absolue Dieu aurait pu ou pourrait faire (33). Ainsi, la théologie est conduite d'une part à repenser son rapport aux disciplines profanes, philosophie et science, et d'autre part à réexaminer le rapport entre puissance absolue et puissance ordonnée.

À ce sujet les spéculations sur les mondes possibles vont se développer, indépendamment de la physique aristotélicienne, puisque celle-ci correspond au créé que nous connaissons, et par conséquent se trouve être totalement contingente. Jean Buridan et Nicolas Oresme, au XIV $V^{\mathrm{e}}$ siècle, imagineront des mondes dotés d'autres lois

(32) Voir BoulnoIs, op. cit., p. 46 sq.

(33) Voir, L. BIANCHI, E. Rand, Vérités dissonantes. Aristote à la fin du Moyen Âge, Fribourg/Paris, Éd. Universitaires de Fribourg/Cerf, 1993, p. 65. 
physiques pour résoudre des problèmes non encore résolus de la physique aristotélicienne (34). Les notions de monde et de nature commencent à être dissociées, alors qu'elles étaient associées chez

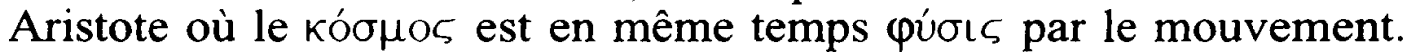
Dorénavant le monde est la totalité de ce qui est, au moins dans ce qui nous est accessible, mais cette totalité peut être relative s'il existe plusieurs mondes. La nature est perçue comme ce qui est structuré par des lois (35).

À partir de la fin du XIII" siècle les discussions théologiques vont d'ailleurs aboutir à refonder le cadre conceptuel de l'approche du monde réel (36). Car, la théologie a fait s'ouvrir le cadre rigide d'Aristote et c'est du côté de la théologie que se développe la question de la pluralité des mondes en lien avec les débats sur la puissance divine.

D'un côté, l'approfondissement de la notion de puissance en lien avec celle de création à partir de rien aboutit en effet à affirmer que Dieu peut créer autant de matière première qu'il veut (37), porte ouverte à la reconnaissance d'une pluralité réelle des mondes et non plus seulement possible.

De l'autre, le questionnement porte sur le rapport entre puissance ordonnée et puissance absolue. Si plusieurs créations coexistent, cela implique la coexistence de plusieurs ordres différents. Il s'agit d'examiner si Dieu peut vouloir simultanément plusieurs créations. C'est ce qu'envisage Jean Duns Scott en soutenant que la puissance absolue de Dieu peut faire un autre ordre que celui que sa puissance ordonnée a établi. Et cet acte n'est pas désordonné. La liberté pour Dieu consiste à pouvoir toujours choisir entre des possibles simultanés. Dieu, de par sa puissance absolue, n'est pas lié à un ordre donné qui dépend de sa puissance ordonnée et par conséquent il peut faire exister plusieurs ordres différents ou identiques sans contrevenir à sa puissance ordonnée qui établit un monde donné. Chacun des ordres ainsi créés, et donc chacun des mondes, existe en vertu de la puissance ordonnée idoine. Pour Jean Duns Scot nature et ordre se rejoignent, car ce qu'on appelle nature correspond à l'ordre imposé par le Créateur, ordre qui est contingent puisqu'il relève de la puissance ordonnée. Tout ce qui est créé est soumis à l'ordre de la nature imposé par le Créateur. Par

(34) E. Grant, La physique au Moyen Age, Paris, PUF, 1995, p. 97-99.

(35) Bollnois, op. cit., p. 48.

(36) Les spéculations théologiques vont peu à peu contribuer à ruiner la théorie aristotélicienne du mouvement; Duhem, t. 9, p. 408. On s'est en effet rendu compte qu'il fallait considérer la position des corps et leurs distances mutuelles pour comprendre leurs mouvements, c'est-à-dire penser dorénavant les mouvements en termes quantitatifs et non plus seulement qualitatifs. Voir BoulNOIS, p. 256.

(37) Voir par exemple Henri DE GAND, Quaestiones quodlibetales $11, \mathrm{q} .1$ et 13, q. 3 ; Duhem, op. cit., t. 9, p. 374 sq. 
conséquent, aux yeux de la créature, les lois de la nature sont caractérisées par leur nécessité. Mais, vu du côté de Dieu, il n'en est rien, elles sont contingentes. Pour le théologien, l'étude de la nature, comme celle de la morale d'ailleurs, devient celle du contingent et non plus celle du nécessaire (38). Cependant, la pluralité des mondes reste pour Jean Duns Scot une possibilité.

En définitive, à la charnière des $\mathrm{XIII}^{\mathrm{e}}$ et XIV $\mathrm{X}^{\mathrm{e}}$ siècles, on passe de l'idée que plusieurs mondes sont possibles à celle qu'il est possible que plusieurs mondes existent réellement (39).

On tient toujours qu'en vertu des deux livres de la révélation, les Écritures et la nature, l'explicitation théologique de l'affirmation de foi doit rejoindre les connaissances profanes. C'est pourquoi, en tenant compte de la position scientifique dominante d'alors, les théologiens d'avant le XIV ${ }^{\mathrm{e}}$ siècle ont affirmé théologiquement la non existence d'une pluralité de mondes.

En revanche, après 1277 , l'impossibilité d'affirmer que Dieu n'a pu créer qu'un seul monde conduit les théologiens à reconsidérer la relation entre les connaissances profanes et la théologie. Celles-là valent pour le monde que nous appréhendons. Elles sont par conséquent contingentes et ne peuvent prétendre à un statut absolu. La théologie qui s'appuie sur la révélation possède au contraire une portée qui les dépasse et de ce fait excède les capacités de la science et de la philosophie. Dit autrement, la révélation et son explicitation qu'est la théologie se situent à leur horizon. Science et théologie peuvent et doivent contribuer à comprendre la révélation mais celle-ci reste première. C'est pourquoi, en partant de la révélation concernant la toute puissance du Dieu Créateur, la théologie aboutit à la possibilité de l'existence de plusieurs mondes, voire à leur réalité, sans qu'il y ait un fondement dans les connaissances profanes.

Un tel constat pose la question de ce qu'est connaître pour l'être humain. On retrouve le fait que le débat sur la pluralité des mondes est signe d'un enjeu noétique et épistémologique, mais dans une

(38) Jean Duns Scot, Ordinatio 1, d. 44, n' 3, 5 et 13 in Opera omnia, 6, ed. P.A. Sépinski, Civitas Vaticana, 1963, p. 363 sq. Voir aussi BoULNOIS, op. cit., p. 266 et $280 \mathrm{sq}$.

(39) Une telle idée suscite un retentissement profond en cosmologie et en anthropologie, mais aussi en théologie. La place de l'être humain par rapport à ces univers et celle de la révélation et du dessein divin par rapport à d'éventuelles autres créatures intelligentes entraîne des débats qui ont une résonance très contemporaines. S'il existe de telles créatures, elles connaissent Dieu autrement. L'argument est alors qu'une diversité plus grande dans le créé est mieux adaptée pour rendre gloire au Créateur. Voir BoULNOIS, p. 52. La réflexion sur la pluralité des mondes déborde du cadre de la puissance créatrice pour toucher aux domaines de la christologie et de la sotériologie. 
perspective nouvelle due à la doctrine de la création qui introduit la question de la contingence.

\section{Piluralité des mondes, scienc' et théorie de la CONNAissance}

La notion de contingence, entre nécessaire et possible, est au cœur de la compréhension de l'agir divin comme création. Tout monde créé est contingent. Il n'est pas nécessaire et existe parmi une infinité d'autres possibles dont certains auraient été ou sont plus parfaits. Nécessaire, possible, contingent, ces trois catégories de la modalité servent durant tout le Moyen Âge à interpréter la relation entre puissance absolue et puissance ordonnée (40). La contingence exprime la dépendance de ce qui existe par rapport au Créateur. Selon la manière dont elle est pensée, on aboutit à affirmer l'existence d'un seul monde ou de plusieurs. Car, penser la contingence engage un type de relation entre pensée, langage et réalité. Deux auteurs vont nous aider à éclaircir cela.

\section{Un seul univers ordonné par la Sagesse divine: Thomas d'Aquin}

Pour Thomas d'Aquin, il n'y a qu'un seul monde (Summa Theologica p. 1, q. 47, a. 3); même une multiplicité numérique est impossible. Pour le prouver Thomas reprend bien sûr l'argument d'Aristote concernant le mouvement de la terre : une autre terre tomberait sur la terre qui est au centre (ibid.). Mais, Thomas fait plus que de s'appuyer sur la science de son temps. Il réagit en théologien et il assure le fondement de sa réflexion dans la révélation. Le principe du monde est en effet le Créateur transcendant. Le monde dans ce qu'il est résulte de la Sagesse divine ordonnatrice. Il relève de la puissance ordonnée tout comme toute autre organisation possible (Summa Theologica p. 1, q. 25, a. 5 ; De potentia q. 1, a. 3, ad 8 et a. 5). Du coup il ne peut exister qu'une seule organisation et un seul monde réels.

Car, le fait fondamental pour Thomas est que l'univers constitue une unité solidaire (De potentia q. 3, a. 17) (41). L'idée que le monde est ordonné constitue ainsi la base de la position de Thomas d'Aquin : l'unicité du monde tient à son unité perçue comme une structure. L'organisation hiérarchisée des êtres tient à un ensemble de relations qui les lient les uns aux autres. Pour expliciter cette organisation,

(40) Voir A. FUnKENSTEIN, Theology and the Scientific Imagination from the Middle Ages to the Seventeeth Century, Princeton, Princeton University Press, 1986, p. 129 sq.

(41) Voir L.B. GeIger, La participation dans la philosophie de S. Thomas d'Aquin, Paris, Vrin, 1942, p. 379 ; FLnKfnstern, op. cit., p. 131 et 136. 
Thomas reprend à la tradition philosophique la notion de participation. Celle-ci existe selon deux modalités qui permettent de penser le rapport de l'un et du multiple selon les deux traits caractéristiques de toute réalité, à savoir d'un côté son insertion dans une organisation et donc une hiérarchie des êtres, et de l'autre l'unité constitutive de cette réalité par rapport à ses composants (42). Ainsi toute créature existe parce qu'elle participe à l'être de celui qui existe par nature, Dieu. Il s'agit d'une participation directe, sans médiation d'aucune autre réalité (Summa contra Gentiles 2, 21). Mais, l'existence ainsi reçue situe la créature par rapport aux autres selon le schème voulu par la Sagesse divine. Cette disposition est intégrée par l'acte créateur dans la nature même des créatures par la participation selon le deuxième mode au schème divin. De ce fait l'acte créateur qui communique à chaque créature l'existence, selon le premier mode de participation, et la nature selon le second, introduit une hiérarchie parmi les êtres, conforme au dessein divin, en induisant des relations entre les êtres selon leur nature et leurs dispositions.

Or, cette compréhension du réel est intimement dépendante d'une théorie de la connaissance (43). L'être humain connaît une réalité parce qu'elle est présente dans son intellect par le biais d'un acte. Celui-ci rend cette réalité présente dans l'intellect et en provoque la connaissance, acte que Thomas appelle forme (ou espèce) intelligible. Par l'intermédiaire des sens, la réalité produit dans l'intellect cette forme intelligible participée qui unit le sujet qui connaît à la réalité qui est connue (44). Cette forme intelligible, indépendante du sujet connaissant (et donc la même chez tous les sujets), n'a pas d'autre nature que de référer à la réalité dont elle émane (Summa contra Gentiles 3, 51). De même que Thomas d'Aquin pense le monde comme une unité solidaire constituée par les relations existant entre les êtres selon le schème divin, de même il pose l'acte de connaissance comme une unité relationnelle entre le sujet et l'objet. La forme intelligible n'est pas un autre objet qui en serait la représentation, mais le même sous un autre mode d'existence (45). La connaissance atteinte par l'intelligence porte par conséquent sur la réalité elle-même et non sur une représentation (Summa contra Gentiles 2, 75,

(42) Voir GEIGER, op. cit., p. 77-79.

(43) Voir à ce sujet E. GiLSON, Le Thomisme. Introduction à la philosophie de saint Thomas d'Aquin, Paris, Vrin, 1972 ; E.H. WÉBER, La controverse de 1270 à l'Université de Paris et son retentissement sur la pensée de S. Thomas d'Aquin, Paris, Vrin, 1970.

(44) Ibid., p. 237. Voir Summa contra Gentiles 2,73, dans Thomas D'AQuin, Contre Averroès, traduction et notes de Alain de Libera, Paris, Garnier-Flammarion, 1994 , p. 338.

(45) Voir GILSON, op. cit., p. 285-286. 
ad $2 \mathrm{~m})(46)$. La connaissance est ainsi produite par la relation entre l'objet et le sujet. La relation est nécessaire à l'acte de connaissance.

La pensée est donc apte à saisir le réel et à l'exprimer par le langage. Du fait de la situation de l'être humain dans le créé comme microcosme, il est capable de penser le monde. La pensée humaine est ainsi une pensée du global. Toute différente est la perspective de Guillaume d'Ockham.

\section{Pluralité et incomplétude des mondes : Guillaume d'Ockham}

Guillaume d'Ockham distingue deux sens pour le terme monde. Ce peut être l'ensemble de toutes les choses créées, substances et accidents. Dans cette hypothèse, il ne peut exister qu'un seul monde. Mais, on peut comprendre le monde comme un ensemble de choses contenues dans un corps et incluant celui-ci (47). Un monde en ce sens est un ensemble de parties qui sont des substances sans prendre en compte les accidents des substances, en particulier leurs relations. C'est le cas du monde constitué, selon la cosmologie de l'époque, de la terre au centre entourée de neuf cieux. Avec cette définition, Guillaume peut alors conduire sa réflexion sur la pluralité des mondes.

Il montre que Dieu peut créer un monde constitué de substances différentes et même meilleures que celles de ce monde-ci. Ce monde est alors autre spécifiquement et numériquement (48). Les arguments proviennent tous de la tradition théologique, notamment d'Augustin.

Dieu peut faire aussi un autre monde que le nôtre, identique selon les espèces mais distinct numériquement. Guillaume réfute alors les deux arguments provenant d'Aristote et utilisés par ses prédécesseurs, tel Thomas d'Aquin, pour prouver l'unicité du monde. Ces arguments concernent le mouvement naturel d'un corps et la matière informe épuisée par la création de ce monde-ci. Pour écarter le premier Guillaume s'appuie sur un raisonnement développé par d'autres avant lui, comme Richard de Middleton à la fin du XIII ${ }^{e}$ siècle (49). Le feu possède un mouvement du bas vers le haut. Le lieu naturel du feu est le haut, c'est-à-dire le ciel. Un feu allumé en un lieu de la terre se déplace vers le haut cis à la verticale de sa position sur la terre. Si on le transporte ailleurs sur la terre il s'élève toujours vers le haut, mais vers le haut correspondant au lieu où il est dorénavant situé et qui est différent du précédent. Transposant ce raisonnement

(46) Ibid., p. 247 sq.

(47) Guillaume D'OCKHAM, Scriptum in librum primum Sententiarum ordinatio, d. 44, in Opera Theologica, 4, éd. G.I. Etzkorn, F.E. Kelley, , st. Bonaventure (NY), St Bonaventure University, 1979, p. 651.

(48) Ordinatio d. 44, p. 654-655.

(49) Quaestionnes super libros sententiarum 1, d. 44, a. 1, q. 4. 
Guillaume affirme que si la terre de l'autre monde était placée sous le ciel de ce monde-ci, elle se déplacerait vers le lieu numériquement identique à celui vers lequel se déplace la terre de ce monde-ci. Mais, qu'elle soit extérieure à ce monde-ci et sous le ciel de l'autre monde, elle ne se déplacera pas alors vers le centre de ce monde-ci [...] mais elle se déplacera vers le centre de l'autre monde (50). La réfutation $\mathrm{du}$ second argument s'effectue en mettant en avant la puissance divine : Au second argument d'Aristote je réponds en disant que le ciel est composé de la totalité de sa matière déjà faite, mais qu'il $n$ 'est pas composé de la totalité d'une matière qui peut être faite, car Dieu peut faire une autre matière (51).

Dieu peut enfin faire un monde qualitativement différent de ce monde-ci. La différence porte ici sur les accidents des substances (52).

Ces conclusions semblent issues d'une critique des arguments tirés par les théologiens des connaissances profanes, scientifiques et philosophiques. En fait, elles proviennent de l'engagement philosophique de Guillaume qui, à la manière de Thomas d'Aquin mais dans une tout autre direction, porte sur le rapport entre pensée, langage et réalité. Guillaume pense en effet toute réalité comme étant fondamentalement singulière (53). La connaissance s'exprime dans le langage et n'atteint pas la réalité elle-même, bien que langage et pensée la visent tous les deux. La relation de la pensée comme du langage à la réalité tient dans la visée issue de l'expérience et l'alimentant. Pensée et langage sur les choses changent en fonction de l'expérience accumulée en particulier grâce à l'expérience originaire qu'est l'intuition de la réalité dans sa singularité. L'expérience de la réalité produit l'évidence du langage utilisé en dehors duquel la réalité ne peut être connue. Le langage est le substitut de la réalité. La signification exprimée par le langage tient en ce qu'il réfère à la réalité par les signes que sont les concepts auxquels l'esprit humain associe l'expérience mémorisée les concernant. Le langage n'est pas signifiant de la réalité au sens où les concepts en constitueraient une représentation (54). La connaissance est donc fondée sur l'intuition des choses et la science l'est sur l'expérience (55). L'intuition d'une réalité, en tant qu'évidence de son existence ou bien d'être telle, est toujours

(50) Ibid., p. 655-660. Traduction Karger dans BoulNOIS, op. cit., p. 352-353.

(51) Ibid., p. 660. Traduction Karger, dans BouLNOIS, op. cit., p. 355.

(52) Ibid. 1989

(53) Voir P. Alféri, Guillaume d'Ockham. Le singulier, Paris, Éd. de Minuit,

(54) Voir A. DE Muralt, La connaissance intuitive du néant et l'évidence du "je pense ". Le rôle de l'argument de potentia absoluta Dei dans la théorie ockhamienne de la connaissance, Studia Philosophica, 36, Bâle, 1976, p. 133.

(55) Voir GRant, op. cit., p. 40-41. 
possible, car elle est fondée sur la puissance absolue de Dieu qui crée toujours des êtres singuliers (56). Guillaume, on le voit, pousse au maximum les conséquences de l'affirmation d'un Dieu tout puissant pour construire la relation entre pensée, langage et réalité.

Il n'est pas nécessaire de développer davantage ici la philosophie ockhamienne pour voir clairement que le monde ne peut être pensé que comme un ensemble, une collection d'êtres singuliers. Les relations que ces êtres singuliers entretiennent sont liées aux circonstances. De même, celles que l'être humain découvre entre les êtres constituant le monde proviennent de son intelligence mais ne sont pas dans les choses. Selon cette compréhension il n'existe pas de relations nécessaires entre des êtres qui sont contingents (57). Dans cette perspective, par conséquent, l'univers n'est pas une totalité organisée en soi, c'est-à-dire possédant une organisation en propre marquée par des relations inhérentes aux êtres. Cette organisation est elle aussi contingente et accidentelle et elle pourrait donc être tout à fait différente. Un monde est pour Guillaume un ensemble fini d'êtres singuliers qui constitue un quelque chose qui n'est ni une unité, ni un être (58), mais une collection qui reste cependant ouvert ontologiquement à la nouveauté, car des formes nouvelles peuvent apparaître (59). Cette possibilité fait qu'un monde ne saurait être complet au sens d'une totalité exhaustive de ce qui existe au point d'exclure $a$ priori toute autre réalité possible. L'idée d'une pluralité des mondes va avec celle de monde considéré comme incomplet, contingent et singulier. C'est pourquoi il peut y avoir plusieurs mondes réels.

L'accent mis sur la singularité montre que Guillaume d'Ockham développe une pensée du local et non du global à l'opposé de la compréhension thomasienne du réel. L'individu en effet prime sur l'ensemble et le monde lui-même est perçu comme une singularité. On voit apparaître ici la théorie ockhamienne de la connaissance.

En définitive, chez Guillaume d'Ockham comme chez Thomas d'Aquin, les présupposés noétiques conduisent ces deux auteurs à soutenir des positions opposées sur la question de la pluralité des mondes, plus que la condamnation de 1277 . Celle-ci possède néanmoins son importance, car elle engage, comme on l'a vu plus haut, à la fois une redéfinition de la théologie par rapport aux connaissances profanes, science et philosophie, et une réappréciation du rapport de la puissance ordonnée à la puissance absolue du Créateur. Mais,

(56) Voir AlfÉRI, op. cit., 106-109 et 161-172.

(57) Voir AlFÉRI, op. cit., p. 127-134; GRANT, op. cit., p. 39. p. 129.

(58) Voir Expositio super viii libros Physicorum, prol., 17 ; AlfÉRI, op. cit.,

(59) Voir ALFÉRI, op. cit., p. 131-134. 
l'articulation de la pensée au réel et son expression par le langage a un rôle au moins aussi important pour comprendre les engagements ontologiques oppposés de Guillaume et de Thomas et la réponse différente qu'ils apportent à la question de savoir s'il existe un ou plusieurs mondes.

Un autre élément confirme d'ailleurs que la seule condamnation de 1277 n'explique pas tout. Après elle, les théologiens " réalistes " qui développent une noétique différente de Thomas d'Aquin, tout en étant une noétique du global, ont continué d'affirmer l'unicité du monde réel parmi une pluralité toujours possible, comme on l'a vu avec Jean Duns Scot.

L'opposition entre Thomas d'Aquin et Guillaume d'Ockham apparaît d'ailleurs aussi dans leurs conceptions épistémologiques. Dans le système philosophique de Guillaume science et théologie sont deux types de connaissance différents, car ils n'engagent pas le même rapport entre langage et réalité. Même si la science, comme toute connaissance, n'a pas affaire directement aux choses, mais à des termes les signifiant, elle se fonde sur l'enchaînement rigoureux des signes et sur un accès direct à leurs référents dans une expérience intuitive qui vérifie la justesse de la référence (60). Dans le cas de la théologie, c'est impossible, car il n'y a pas d'expérience intuitive de Dieu. Par conséquent, la théologie est purement verbale et ne peut être qu'une théologie négative. Les propositions de la théologie pour les chrétiens sont vraies. C'est-à-dire, explicite Guillaume, on les croit vraies sans les savoir vraies (61). L'epistémologie ockhamienne est aux antipodes de celle de Thomas d'Aquin pour qui la théologie est une science, et même la reine des sciences. Il reprend la notion aristotélicienne de science, une connaissance qui procède par voie démonstrative à partir de principes premiers évidents et connus comme vrais par intuition (62). Or, pour la théologie, ces principes sont les vérités de foi qui ne sont pas évidentes par elles-mêmes, c'est-à-dire accessibles à la raison. Elles sont crues, mais pas connues. Cependant, elles le sont pour les saints qui vivent de la vie bienheureuse et elles sont approchées par ceux qui vivent de la grâce. En effet, ces vérités proviennent de Dieu, et plus particulièrement du Verbe qui est la Vérité (63).

(60) Ibid., p. 430.

(61) Ibid., p. 431

(62) Voir le $\S 1$.

(63) Ces vérités concernent essentiellement l'humanité du Christ Jésus par qui nous avons accès à Dieu et le mystère de Dieu; voir Summa Theologica, p. 2-2, q. 1, a. 8 et le commentaire de H.D. Gardeil dans Saint Thomas d'Aquin, Somme Théo. logique, La théologie, Ia, Prologue et question 1, Paris, Cerf, 1968, p. 116-118. 
En conclusion, le discours sur l'existence d'un monde ou de plusieurs repose, comme tout discours rationnel, sur des présupposés noétiques et épistémologiques qui se laissent découvrir par certains termes qui caractérisent précisément la théorie de la connaissance sous-jacente au discours. Ces termes sont chez Thomas d'Aquin relation, unité et participation. Ils engagent une pensée du global où la pensée participe du réel et l'exprime dans le langage et où les relations entre les êtres correspondent à la structure de la pensée que l'intelligence produit à leur sujet. Chez Guillaume, au contraire, les termes de singulier, de signe et d'intuition engagent une pensée du local où la pensée et le langage réfèrent tous deux au réel mais de manière différente. Le premier, par des signes issus de l'intuition et combinés entre eux grâce à l'expérience accumulée, le second par les signes-concepts élaborés par l'abstraction et employés dans le discours rationnel pour parler du réel.

En définitive, les présupposés conceptuels se rapportant à ce que sont respectivement l'acte de connaître et la science sont tout aussi importants que la réponse que l'on donne aux questions concernant le réel, car ils dessinent les perspectives et donc les questionnements que ces systèmes de pensée permettent. La question se pose alors de savoir si, pour répondre à une question, comme la pluralité des mondes envisagée ici, il ne vaut pas mieux recourir à plusieurs systèmes de pensée reposant sur des noétiques et des épistémologies différentes. Dans cette hypothèse, il est nécessaire de voir comment différentes manières de connaitre peuvent se raccorder, voire se recouvrir au moins partiellement. Connaître ne consisterait plus alors à dessiner une carte exhaustive de la réalité envisagée où figureraient toutes les réalités connues grâce à la conceptualisation mise en œuvre. Connaître consisterait à réaliser un ensemble de cartes de conception et d'échelle différentes, se recouvrant plus ou moins, comportant des indications, issus de la réflexion selon la perspective d'une discipline précise, les liens entre les cartes n'étant pas toujours immédiats mais passant par des procédures de corrélation de l'une à l'autre. C'est une démarche qui est utilisée en science aujourd'hui, par exemple en logique et en physique, le raccordement étant plus élaboré dans le premier cas que dans le second. Il serait intéressant de s'interroger si cette démarche pourrait trouver une application dans le traitement de questions touchant à des disciplines aussi différentes que science, philosophie et théologie. 